\title{
Metabolomic changes associated with frontotemporal lobar degeneration syndromes
}

\author{
Alexander G. Murley ${ }^{1,3}$ (D) P. Simon Jones ${ }^{1} \cdot$ lan Coyle Gilchrist ${ }^{4} \cdot$ Lucy Bowns $^{1} \cdot$ Julie Wiggins $^{1}$. \\ Kamen A. Tsvetanov ${ }^{1}$. James B. Rowe $\mathrm{e}^{1,2,3}$
}

Received: 10 February 2020 / Revised: 2 April 2020 / Accepted: 3 April 2020 / Published online: 10 April 2020

(c) The Author(s) 2020

\begin{abstract}
Objective Widespread metabolic changes are seen in neurodegenerative disease and could be used as biomarkers for diagnosis and disease monitoring. They may also reveal disease mechanisms that could be a target for therapy. In this study we looked for blood-based biomarkers in syndromes associated with frontotemporal lobar degeneration.

Methods Plasma metabolomic profiles were measured from 134 patients with a syndrome associated with frontotemporal lobar degeneration (behavioural variant frontotemporal dementia $n=30$, non fluent variant primary progressive aphasia $n=26$, progressive supranuclear palsy $n=45$, corticobasal syndrome $n=33$ ) and 32 healthy controls.

Results Forty-nine of 842 metabolites were significantly altered in frontotemporal lobar degeneration syndromes (after false-discovery rate correction for multiple comparisons). These were distributed across a wide range of metabolic pathways including amino acids, energy and carbohydrate, cofactor and vitamin, lipid and nucleotide pathways. The metabolomic profile supported classification between frontotemporal lobar degeneration syndromes and controls with high accuracy (88.196.6\%) while classification accuracy was lower between the frontotemporal lobar degeneration syndromes (72.1-83.3\%). One metabolic profile, comprising a range of different pathways, was consistently identified as a feature of each disease versus controls: the degree to which a patient expressed this metabolomic profile was associated with their subsequent survival (hazard ratio 0.74 [0.59-0.93], $p=0.0018$ ).

Conclusions The metabolic changes in FTLD are promising diagnostic and prognostic biomarkers. Further work is required to replicate these findings, examine longitudinal change, and test their utility in differentiating between FTLD syndromes that are pathologically distinct but phenotypically similar.
\end{abstract}

Keywords Frontotemporal lobar degeneration $\cdot$ Frontotemporal dementia $\cdot$ Primary progressive aphasia $\cdot$ Progressive supranuclear palsy $\cdot$ Corticobasal syndrome $\cdot$ Metabolomics

\section{Introduction}

Electronic supplementary material The online version of this article (https://doi.org/10.1007/s00415-020-09824-1) contains supplementary material, which is available to authorized users.

Alexander G. Murley

am2505@medschl.cam.ac.uk

1 Department of Clinical Neurosciences, University of Cambridge, Cambridge, UK

2 MRC Cognition and Brain Sciences Unit, University of Cambridge, Cambridge, UK

3 Cambridge University Hospitals NHS Foundation Trust, Cambridge, UK

4 Norfolk and Norwich University Hospitals NHS Foundation Trust, Norwich, UK
Frontotemporal lobar degeneration (FTLD) causes a wide spectrum of syndromes including the behavioural and language variants of frontotemporal dementia (bvFTD, PPA, respectively), progressive supranuclear palsy (PSP) and corticobasal syndrome (CBS) [1, 2]. Accurate early diagnosis is challenging, due in part to the specialist clinical skills and imaging resources required. There is therefore a pressing need for FTLD biomarkers. Such biomarkers may also facilitate diagnosis, clinical trials monitoring and reveal disease mechanisms as a target for therapy. Metabolomics is one approach, that has identified abnormal metabolic pathways in other neurodegenerative diseases including Alzheimer's, Huntington's and Parkinson's diseases [3-6]. We therefore 
examined blood-based metabolic biomarkers in four clinical syndromes associated with FTLD. We studied the four syndromes together, in view of their potential commonalities in clinical and neuropathological features $[1,7,8]$.

Metabolic pathways are likely to be altered in FTLD. For example, genomic studies of FTLD syndromes have identified genetic polymorphisms implicated in protein synthesis, packaging and breakdown, as well as immune functions and myelin structure [9-13]. However, there is limited evidence on metabolomic abnormalities in FTLD: the cerebrospinal fluid in FTD shows a panel of metabolites could differentiate FTD from controls and Alzheimer's disease [14], while hypertriglyceridemia and hypoalphalipoproteinemia have been reported in bvFTD [15]. Several metabolites were of particular interest. For example, phosphatidylserine has been proposed as a pro-apoptotic marker in pre-clinical neuronal models of tauopathies [16, 17], and might therefore be abnormal in the clinical tauopathies within FTLD. Amino acids such as the neurotransmitter serotonin are also deregulated in FTLD [18, 19], while secondary changes in dietary preferences, weight and exercise associated with FTLD may influence carbohydrate metabolism [20-23].

This study had three aims. First, to identify which biochemicals and their associated metabolite pathways are abnormal in each of four FTLD syndromes. Second, to test the accuracy of metabolite profiles in classifying patients versus healthy controls. Third, to test whether metabolomics changes are indicative of prognosis. We predicted that a wide range of metabolic pathways would be abnormal in FTLD, supporting accurate classification between FTLD syndromes and controls; but phenotypic and pathological similarities would reduce the accuracy of differential diagnosis between the FTLD syndromes.

\section{Materials and methods}

\section{Study participants}

Patients were recruited from the Cambridge Centre for Frontotemporal Dementia and Related Disorders and met the clinical diagnostic criteria for either behavioural variant frontotemporal dementia [24], non-fluent variant primary progressive aphasia [25], progressive supranuclear palsy Richardson's syndrome [26] or corticobasal syndrome [27]. Healthy controls had no neurological or psychiatric disease, but were not screened by genetics or biomarkers to exclude asymptomatic neuropathologies. The study was approved by the local ethics committee and all participants gave informed consent or, if lacking mental capacity, through a consultee process according to UK law. 134 patients (30 bvFTD, 26 nfvPPA, 45 PSP, 33 CBS) and 32 healthy controls participated. Plasma was obtained by centrifugation of whole blood and stored at $-80^{\circ} \mathrm{C}$ until analysis.

\section{Metabolite detection and quantification}

Biochemical identification and quantification were performed by Metabolon Inc (www.metabolon.com) for all samples at a single timepoint. Samples were analysed with ultra-high performance liquid chromatography and tandem mass spectrometry, optimised for basic and acidic species. Biochemicals were then identified by comparison of the ion features of each sample to a reference library of compounds and grouped into sub and super pathways, corresponding to metabolite pathways [28]. For a full list of the metabolic pathways and their constituent biochemicals measured in this study see Appendix 1.

\section{Statistical analysis}

Our statistical analysis pipeline is summarised in Fig. 1. First, we used independent two-sample $t$ tests to compare the age distributions of the FTLD and control groups. A Chi-squared test with Yates correction was used to compare sex between groups. In the metabolite dataset missing values implied a result below the limit of detection in that individual. We excluded metabolites if they were missing in more than half of the participants. Remaining missing values were replaced by half of the minimum positive value of that variable. We also removed metabolites from exogenous metabolic pathways, including known drugs and drug pathways, before further analysis. All metabolite concentrations were scaled to unit variance (i.e., normalised to $z$ scores) [29].

Univariate statistical tests were then used to compare individual metabolite differences between groups. We used a generalised linear model on each metabolite, with age and sex as covariates, to compare the FTLD and control groups. An FDR corrected $p$ value threshold of 0.01 used to determine statistical significance (using the 'mafdr' function in MATLAB). Bonferroni correction is also presented, while noting that non-independence of metabolites is likely to make this method overly conservative. The fold change for each metabolite was calculated by dividing the mean disease and control values of unscaled data.

A two-level principal component analysis (PCA) was used to explore the diseases' effect on each metabolite pathway. We used this two-level approach to reduce dimensionality whilst preserving the metabolite pathways structure of the dataset, with the parsimonious representation of all metabolic pathways in the comparisons between groups. At the first level, we performed a 'local PCA' on the metabolites in each subpathway, to identify components that best explained the variance in that pathway. Ninety-one local principal component analyses were run in total, one for each 


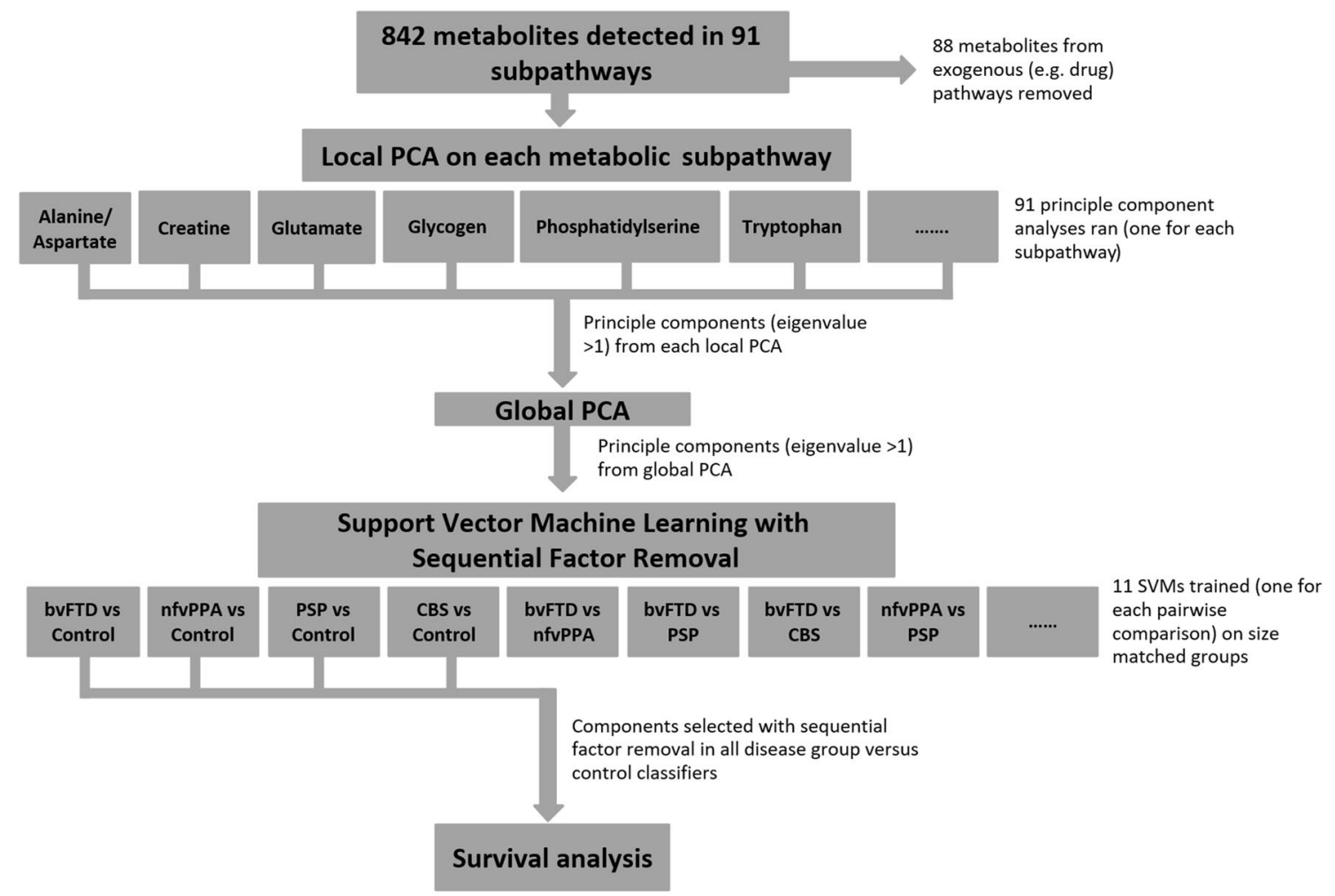

Fig. 1 Summary of the analysis pipeline. From a total of 842 metabolites, a principal component analysis (PCA) was run on the metabolites in each of 91 subpathways. All components with an eigenvalue greater than 1 were entered into a global PCA. The subject-specific weights of the principal components from this PCA were used as fea-

metabolite subpathway. Within each subpathway, we used the Kaiser criteria to select components with an eigenvalue greater than one. To assess which metabolite pathways are affected in FTLD we used independent two-sample $t$ tests to compare scores for each local PCA component. An FDR corrected $p$ value threshold of 0.01 was used to determine statistical significance. At the second level, we performed a 'global PCA'. This was global in the sense of examining metabolite variance across all subpathways, including all the components with an eigenvalue greater than one from all local PCAs.

Next we tested the ability of the global PCA components to classify FTLD syndromes. We trained pairwise linear support vector machines using the subject specific weightings for components output from the global PCA. A total of eleven SVMs were trained, to discriminate between each of the five groups, and to compare all FTLD syndromes jointly versus healthy controls. Prior to training, component loading values were rescaled from -1 to 1 . Groups were sizematched by randomly sampling cases from the larger of the groups to match the size of the smaller group.

We used backwards sequential feature selection using the 'sequentialfs' function in MATLAB to identify the tures for support vector machines, using $k$-fold cross-validation and recursive feature elimination. Components selected by recursive feature elimination were then used as predictors for the survival analysis (cox proportional hazards regression with age, gender and FTLD subgroup as covariates)

components that best predicted disease, as follows. Starting with the full dataset, components were sequentially removed until classifier accuracy decreased. SVM accuracy and factor selection were validated with tenfold cross validation. In each iteration, the training and test data subsets were kept separate. Random case sampling, SVM training and sequential feature selection were repeated 10 times and the mean accuracy overall partitions were calculated. Only the components selected in all repetitions are reported. With small sample sizes, $k$-fold cross-validation minimises the bias of within-sample cross-validation [30]. The reported accuracy from each SVM is the mean accuracy from all SVMs trained for each pairwise comparison. Out of sample cross-validation is provided indirectly by comparison of the components that were consistent contributors to accurate classification for each of the four syndromes versus controls.

Next, we investigated the relationship between FTLDassociated metabolome and survival. Survival analysis was performed with Cox proportional hazards regression. Only components selected by sequential feature selection in all disease versus control SVMs were used as predictor variables. Age, gender and FTLD-group were entered as covariates. SVM analyses were performed using LIBSVM 
in MATLAB R2018b (MathWorks) [31]. Other statistical tests used MATLAB R2018b (Mathworks, USA).

\section{Results}

Table 1 summarises the clinical groups. There were significant differences between FTLD (all diseases combined) and control samples in forty-nine out of 842 metabolites detected (two sample $t$ test, FDR $p<0.01$ ). The statistical significance of each metabolite is plotted against fold-change in Fig. 2a. These metabolites did not cluster in one pathway but were distributed across a wide range of metabolic pathways. These included sixteen amino acid, seven energy and carbohydrate, three cofactor and vitamin, sixteen lipid, three nucleotide and four xenobiotic pathways (Table 2).
Twenty-six of these metabolites remained significant with family wise error correction (Bonferroni corrected $p<0.01$ ), distributed across a wide range of pathways.

To assess differences in metabolic pathways, as opposed to individual metabolites, we compared the component loadings of principal component analyses on the metabolites in each pathway. Principal component analysis on each of 91 sub-pathways yielded 230 individual components. The component scores on twelve sub-pathways were significantly different between FTLD and controls (two sample $t$ test, FDR $p<0.01)$. These included widespread changes in the metabolome including amino acid (creatine, glutamate, glycine, serine, threonine, methionine, cysteine, taurine, polyamine and tryptophan), carbohydrate (amino sugar and glycogen) and lipid (fatty acid, lysoplasmalogen, mevalonate, monoacylglycerol and phospholipid) pathways.

Table 1 Demographic and clinical summary metrics of study participants

\begin{tabular}{|c|c|c|c|c|c|c|c|}
\hline & FTLD (all subgroups) & bvFTD & nfvPPA & PSP & CBS & Control & $\begin{array}{l}p \text { value } \\
\text { (FTLD vs } \\
\text { control) }\end{array}$ \\
\hline Number & 134 & 30 & 26 & 45 & 33 & 32 & \\
\hline Mean age at blood test (SD) & $70.36(8.21)$ & $64.51(7.17)$ & $72.00(7.66)$ & $72.9(8.06)$ & $70.91(7.04)$ & $68.73(9.03)$ & ns \\
\hline$\%$ Male & 50 & 50 & 38 & 62 & 55 & 56 & ns \\
\hline $\begin{array}{l}\text { Symptom onset to study (in years) } \\
\text { (SD) }\end{array}$ & $4.86(2.86)$ & $5.56(2.91)$ & $4.59(2.1)$ & $4.71(3.12)$ & $4.68(2.84)$ & - & ns \\
\hline Diagnosis to study (in years) (SD) & $1.52(1.73)$ & $2.0(2.11)$ & $1.64(1.46)$ & $1.04(1.39)$ & $1.68(1.82)$ & - & ns \\
\hline Mean ACE-R $(<100)(\mathrm{SD})$ & $62(27)$ & $52(30)$ & $61(29)$ & $72(22)$ & $61(29)$ & - & 0.009 \\
\hline Mean CBI $(<180)(\mathrm{SD})$ & $61(28)$ & $83(26)$ & $39(32)$ & $56(31)$ & $66(34)$ & - & $<0.001$ \\
\hline Mean PSP-RS $(<100)(S D)$ & - & - & - & $43(15)$ & - & - & NA \\
\hline
\end{tabular}

$p$ values are the result of ANOVA across rows for all FTLD subgroups and controls (where applicable), except \% Male where a Chi squared test was used: $\mathrm{ns}=p>0.05$

ACER Addenbrookes Cognitive Examination—Revised, CBI Cambridge Behavioural Inventory-Revised, PSP-RS Progressive Supranuclear Palsy Rating Scale

Fig. 2 Metabolomic alterations in FTLD syndromes. Volcano plot of log-fold change in each metabolite for the contrast of FTLD vs control, and their significance (log-FDR corrected $p$ value). Metabolites are colour coded by superpathway. The horizontal line marks $p=0.01$ significance. The significant metabolites above this line, both increased and decreased, come from each the major metabolic pathways

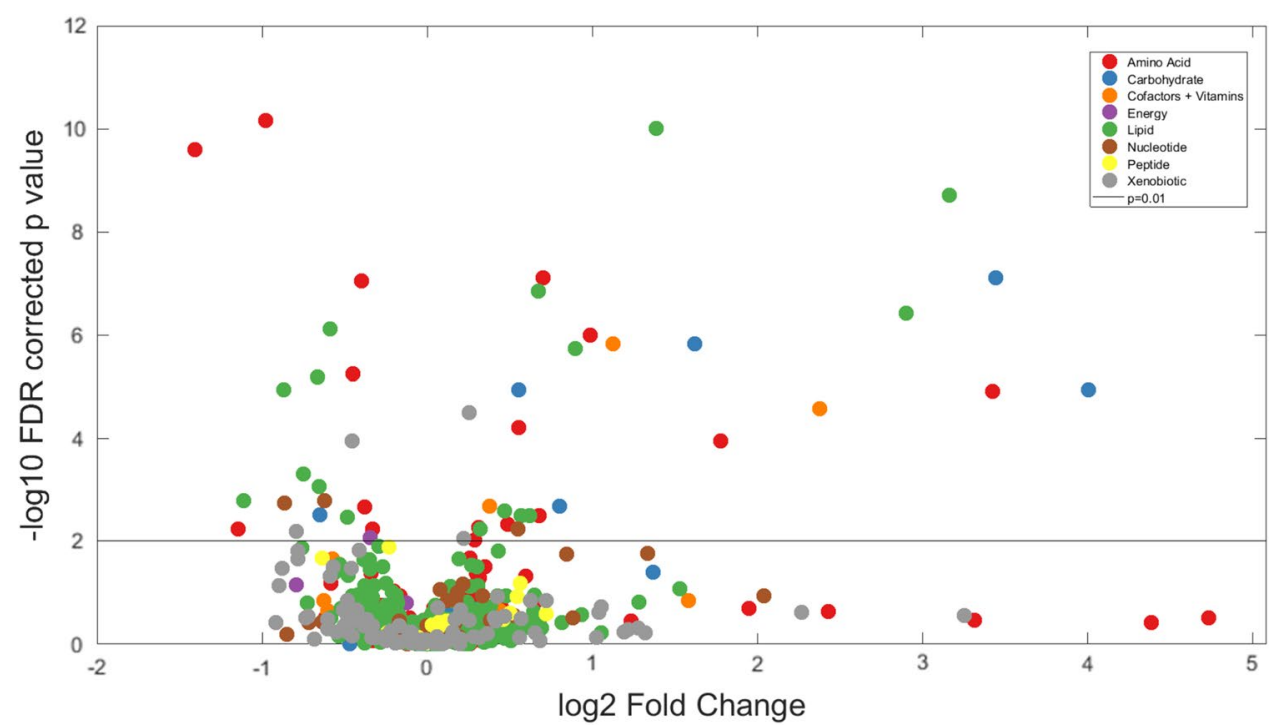


Table 2 Table of metabolites that were significantly different in combined FTLD syndromes, compared to healthy controls

\begin{tabular}{|c|c|c|c|c|c|}
\hline Metabolite name & Subpathway & Superpathway & Fold change & $p$ value (FDR) & $p$ value (uncorr) \\
\hline Guanidinoacetate & Creatine & Amino acid & 0.73 & $5.73 \mathrm{E}-06$ & $1.14 E-07$ \\
\hline Beta-citrylglutamate & Glutamate & Amino acid & 1.47 & $6.33 \mathrm{E}-05$ & $1.93 E-06$ \\
\hline 1-Pyrroline-5-carboxylate & Glutamate & Amino acid & 1.60 & $3.14 \mathrm{E}-03$ & $1.58 \mathrm{E}-04$ \\
\hline 2-Aminobutyrate & Glutathione & Amino acid & 0.77 & $2.19 \mathrm{E}-03$ & $9.57 \mathrm{E}-05$ \\
\hline Sarcosine & Glycine/serine/threonine & Amino acid & 0.76 & $9.13 \mathrm{E}-08$ & $8.48 \mathrm{E}-10$ \\
\hline 2-Methylserine & Glycine/serine/threonine & Amino acid & 0.51 & $7.02 \mathrm{E}-11$ & $9.31 E-14$ \\
\hline$N$-Acetylmethionine & Methionine/cysteine/sam/taurine & Amino acid & 1.22 & $9.61 \mathrm{E}-03$ & $6.25 \mathrm{E}-04$ \\
\hline Alpha-ketobutyrate & Methionine/cysteine/sam/taurine & Amino acid & 0.38 & $2.53 \mathrm{E}-10$ & $1.01 \mathrm{E}-12$ \\
\hline Hypotaurine & Methionine/cysteine/sam/taurine & Amino acid & 1.98 & $1.03 \mathrm{E}-06$ & $1.51 \mathrm{E}-08$ \\
\hline Taurine & Methionine/cysteine/sam/taurine & Amino acid & 1.63 & $7.68 \mathrm{E}-08$ & $5.14 \mathrm{E}-10$ \\
\hline Spermidine & Polyamine & Amino acid & 3.42 & $1.12 \mathrm{E}-04$ & $3.69 \mathrm{E}-06$ \\
\hline 5-Methylthioadenosine (MTA) & Polyamine & Amino acid & 1.24 & $5.34 \mathrm{E}-03$ & $2.91 \mathrm{E}-04$ \\
\hline Tryptophan betaine & Tryptophan & Amino acid & 0.45 & $5.90 \mathrm{E}-03$ & $3.50 \mathrm{E}-04$ \\
\hline Serotonin & Tryptophan & Amino acid & 10.71 & $1.22 \mathrm{E}-05$ & $3.23 \mathrm{E}-07$ \\
\hline Homoarginine & Urea cycle; arginine/proline & Amino acid & 0.79 & $5.78 \mathrm{E}-03$ & $3.30 \mathrm{E}-04$ \\
\hline pro-Hydroxy-pro & Urea cycle; arginine/proline & Amino acid & 1.40 & $4.77 \mathrm{E}-03$ & $2.53 \mathrm{E}-04$ \\
\hline$N$-Acetylneuraminate & Aminosugar & Carbohydrate & 1.47 & $1.14 \mathrm{E}-05$ & $2.83 \mathrm{E}-07$ \\
\hline $\mathrm{N}$-Acetylglucosaminylasparagine & Aminosugar & Carbohydrate & 1.74 & $2.10 \mathrm{E}-03$ & $8.93 \mathrm{E}-05$ \\
\hline Maltotetraose & Glycogen & Carbohydrate & 16.02 & $1.14 \mathrm{E}-05$ & $2.58 E-07$ \\
\hline Maltotriose & Glycogen & Carbohydrate & 10.87 & $7.68 \mathrm{E}-08$ & $6.11 E-10$ \\
\hline Maltose & Glycogen & Carbohydrate & 3.08 & $1.50 \mathrm{E}-06$ & $2.58 \mathrm{E}-08$ \\
\hline Pyruvate & Glycolysis/gluconeogenesis/pyruvate & Carbohydrate & 0.64 & $3.13 \mathrm{E}-03$ & $1.45 \mathrm{E}-04$ \\
\hline Nicotinamide & Nicotinate/nicotinamide & Cofactors/vitamins & 2.18 & $1.50 \mathrm{E}-06$ & $2.48 \mathrm{E}-08$ \\
\hline $\begin{array}{l}\text { Adenosine 5'-diphosphoribose (ADP- } \\
\text { ribose) }\end{array}$ & Nicotinate/nicotinamide & Cofactors/vitamins & 5.19 & $2.70 \mathrm{E}-05$ & $7.52 \mathrm{E}-07$ \\
\hline Flavin adenine dinucleotide (FAD) & Riboflavin & Cofactors/vitamins & 1.30 & $2.10 \mathrm{E}-03$ & $8.87 \mathrm{E}-05$ \\
\hline Succinate & TCA cycle & Energy & 0.79 & $8.55 \mathrm{E}-03$ & $5.33 \mathrm{E}-04$ \\
\hline Stearamide & Fatty acid/amide & Lipid & 0.72 & $3.41 \mathrm{E}-03$ & $1.76 \mathrm{E}-04$ \\
\hline Pristanate & Fatty acid/branched & Lipid & 0.63 & $8.63 \mathrm{E}-04$ & $3.09 \mathrm{E}-05$ \\
\hline Maleate & Fatty acid/dicarboxylate & Lipid & 0.55 & $1.14 \mathrm{E}-05$ & $2.88 \mathrm{E}-07$ \\
\hline Glycerol 3-phosphate & Glycerolipid & Lipid & 0.66 & $7.69 \mathrm{E}-07$ & $1.02 \mathrm{E}-08$ \\
\hline 1-(1-Enyl-palmitoyl)-GPE $(P-16: 0)^{*}$ & Lysoplasmalogen & Lipid & 1.25 & $5.77 \mathrm{E}-03$ & $3.21 \mathrm{E}-04$ \\
\hline Heptanoate $(7: 0)$ & Medium chain fatty acid & Lipid & 1.86 & $1.85 \mathrm{E}-06$ & $3.43 \mathrm{E}-08$ \\
\hline 10-Undecenoate $(11: 1 \mathrm{n} 1)$ & Medium chain fatty acid & Lipid & 0.63 & $6.67 \mathrm{E}-06$ & $1.42 \mathrm{E}-07$ \\
\hline 1-Palmitoleoylglycerol (16:1) & Monoacylglycerol & Lipid & 0.46 & $1.65 \mathrm{E}-03$ & $6.35 \mathrm{E}-05$ \\
\hline 1-Linoleoylglycerol (18:2) & Monoacylglycerol & Lipid & 0.59 & $4.87 \mathrm{E}-04$ & $1.68 \mathrm{E}-05$ \\
\hline 1-Stearoyl-2-oleoyl-GPS (18:0/18:1) & Phosphatidylserine (PS) & Lipid & 8.94 & $1.95 \mathrm{E}-09$ & $1.03 \mathrm{E}-11$ \\
\hline $\begin{array}{l}\text { 1-Stearoyl-2-arachidonoyl-GPS } \\
\quad(18: 0 / 20: 4)\end{array}$ & Phosphatidylserine (PS) & Lipid & 7.47 & $3.82 \mathrm{E}-07$ & $4.56 \mathrm{E}-09$ \\
\hline Choline phosphate & Phospholipid & Lipid & 1.59 & $1.42 \mathrm{E}-07$ & $1.50 \mathrm{E}-09$ \\
\hline Phosphoethanolamine & Phospholipid & Lipid & 2.61 & $9.92 \mathrm{E}-11$ & $2.63 \mathrm{E}-13$ \\
\hline Sphinganine & Sphingolipid & Lipid & 1.53 & $3.14 \mathrm{E}-03$ & $1.55 \mathrm{E}-04$ \\
\hline Sphingosine & Sphingolipid & Lipid & 1.38 & $2.57 \mathrm{E}-03$ & $1.16 \mathrm{E}-04$ \\
\hline $\begin{array}{l}\text { Lactosyl- } N \text {-behenoyl-sphingosine } \\
\qquad(18: 1 / 22: 0)\end{array}$ & Sphingolipid & Lipid & 1.48 & $3.14 \mathrm{E}-03$ & $1.50 \mathrm{E}-04$ \\
\hline N1-Methylinosine & Purine/(hypo)xanthine/inosine containing & Nucleotide & 1.46 & $5.90 \mathrm{E}-03$ & $3.52 \mathrm{E}-04$ \\
\hline Dihydroorotate & Pyrimidine/orotate containing & Nucleotide & 0.55 & $1.80 \mathrm{E}-03$ & $7.18 \mathrm{E}-05$ \\
\hline 2'-Deoxyuridine & Pyrimidine/uracil containing & Nucleotide & 0.65 & $1.62 \mathrm{E}-03$ & $6.02 \mathrm{E}-05$ \\
\hline Benzoate & Benzoate & Xenobiotics & 0.73 & $1.12 \mathrm{E}-04$ & $3.72 \mathrm{E}-06$ \\
\hline Iminodiacetate (IDA) & Chemical & Xenobiotics & 1.19 & $3.13 \mathrm{E}-05$ & $9.15 \mathrm{E}-07$ \\
\hline Thioproline & Chemical & Xenobiotics & 1.16 & $8.69 \mathrm{E}-03$ & $5.53 \mathrm{E}-04$ \\
\hline 1-Methylxanthine & Xanthine & Xenobiotics & 0.58 & $6.34 \mathrm{E}-03$ & $3.87 \mathrm{E}-04$ \\
\hline
\end{tabular}

$p$ value columns show the $p$ value for a generalised linear model between FTLD and controls with age and sex as covariates. $p$ values in the uncorrected column in bold indicate survival after Bonferroni correction (equivalent to uncorrected threshold $p<1.33 \mathrm{e}^{-5}$ ) 
We then tested the efficacy of metabolomics as a diagnostic biomarker for FTLD (Table 3). Linear support vector machines with sequential feature selection using all 50 principal components from the global PCA as predictor variables accurately distinguished FTLD from controls (92.5\%) and individual FTLD syndromes from controls (bvFTD 96.67\% nfvPPA $88.08 \%$ PSP 95.78\% CBS 95.16\%). Accuracy was less among FTLD syndromes. BvFTD classification accuracy from nfvPPA (82.00\%), PSP (81.33\%) and CBS $(83.33 \%)$ was better than PSP, CBS and nfvPPA. This was even lower in separating nfvPPA from PSP $(79.52 \%)$ or CBS $(0.72 \%)$ and PSP from CBS (78.6\%).

Sequential feature selection, by removing components that did not contribute to SVM accuracy, identifies the components that best separated the two groups. Only two or three components were selected for each disease vs control classifier. One principal component was selected in every comparison between disease group and controls (component 3 ). From the between syndrome classifications, multiple additional components were consistently selected (up to 6 in the bvFTD vs CBS comparison). For the nfvPPA vs CBS classifier accuracy no components were consistently selected.

Component 3, from the global PCA of all metabolite pathways, was selected by sequential feature selection in every disease vs control classifier. This means the metabolites contributing to this component were consistently important in separating disease groups from controls. All but two healthy controls positively loaded onto this component while the loadings in the FTLD syndromes varied (Fig. 3a). Component 3 represented metabolites from a wide range of pathways (Fig. 3b, full list of subpathway loadings in Appendix 2). Sub-pathways with high positive loading onto component 3 included phospholipid and other lipid pathways, haemoglobin and the carbohydrate glycogen metabolism pathway. Subpathways with high negative

Table 3 Matrix of average classification accuracy of the support vector machines' classification between groups (percentage total correct classification)

\begin{tabular}{llllll}
\hline & bvFTD & nfvPPA & PSP & CBS & Control \\
\hline bvFTD & 86 & 82 & 81 & 83 & 97 \\
nfvPPA & 82 & 80 & 76 & 72 & 88 \\
PSP & 81 & 76 & 83 & 79 & 96 \\
CBS & 83 & 72 & 79 & 82 & 95 \\
Control & 97 & 88 & 96 & 95 & 93 \\
\hline
\end{tabular}

Groups were sized matched for each classifier (see "Materials and methods"). The diagonal values represent the classification accuracy for that disease group against all other groups combined. Classification accuracy is high in each FTLD syndrome compared with healthy controls, but lower when classifying between FTLD syndromes

$b v F T D$ behavioural variant frontotemporal dementia, nfvPPA nonfluent variant primary progressive aphasia, $P S P$ progressive supranuclear palsy Richardson's syndrome, $C B S$ corticobasal syndrome loading onto component 3 included certain fatty-acid pathways and amino acid pathways including leucine, valine, tryptophan, glycine, serine, threonine, methionine, cysteine and taurine metabolism.

We next tested component 3 as a prognostic biomarker (in patients only) using Cox proportional hazards regression using age, gender, disease groups and component 3 and days from blood test to death. The standardised individual participant loadings on component 3 were significantly associated with time to death [hazard ratio $0.74(0.59-0.93)$, $p=0.0018]$. To illustrate this effect, we plotted separately the patients with high $(z$ score $>1)$, medium $(z$ score between 1 and -1$)$ and low $(z$ score $<1)$ values on this component (Fig. 4).

\section{Discussion}

Our results show that multiple metabolic pathways are changed in patients with clinical syndromes associated with frontotemporal lobar degeneration. One particular profile of metabolic change (here identified as component 3 ) was consistently identified as a feature of each disease versus controls, and the degree to which a patient expressed this metabolomic profile was correlated with subsequent survival. The metabolic changes in FTLD were not confined to a single pathway but were found across carbohydrates, lipids, amino acids, and peptide pathways. The identification of a blood-based metabolic index of FTLD and survival may in future assist prognostication and clinical trial design. However replication is required and we acknowledge that our results do not determine whether these abnormalities result from aetiopathogenic processes or environmental sequelae of neurodegenerative disease. Replication in independent cohorts and the analysis of longitudinal change will be important extensions of this work. In the following, we discuss the metabolic changes, in turn, their potential utility for diagnosis and prognosis, and the study's limitations.

Our first aim was to identify metabolic markers of FTLD. Several of the metabolite differences in FTLD implicate carbohydrate metabolism and energy pathways. Maltose and maltose metabolites, elevated in our FTLD groups, are primary disaccharides in the human diet. This result may be due to the altered dietary preferences, appetite, weight change and exercise associated with FTLD [20-23]. However, it may also be due to endogenous changes in energy metabolism and storage. Pyruvate and succinate, both key components of the TCA cycle, were low in FTLD despite the raised polysaccharides levels. Glycerol-3-phosphate, which has an important role in reoxidisation of NADH, was also low. These abnormalities reflect altered glucose uptake and metabolic dysfunction, which is of particular interest in view of in vivo PET imaging of FTLD where abnormal 
Fig. 3 a Individual loading onto component 3 , by group. b Subpathways loading on component 3
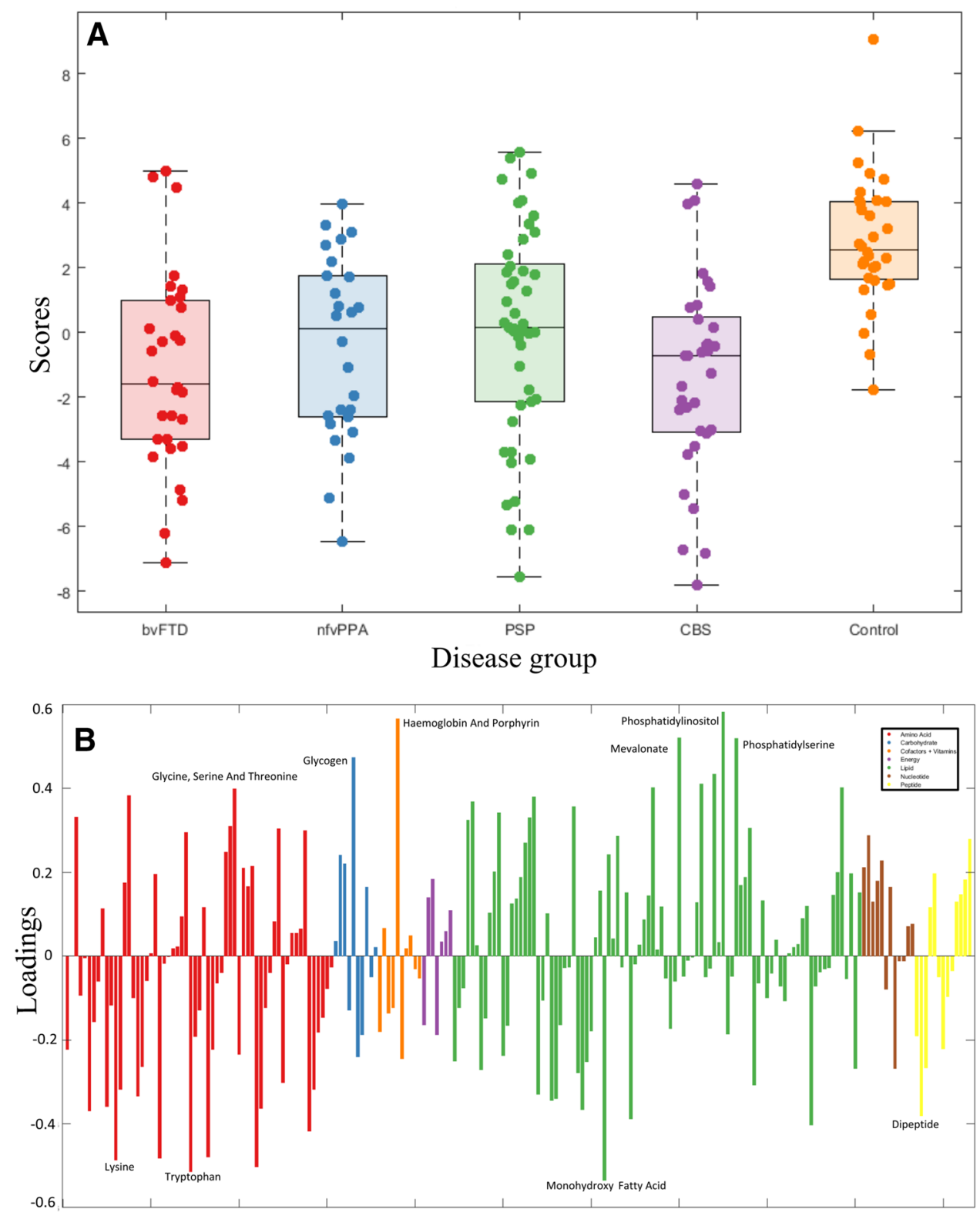

glucose metabolism often precedes neuronal loss and atrophy [32-34].

The amino acid differences could also be attributed to defective energy metabolism. For example, glucogenic amino acid metabolites, including alpha-ketobutyrate, 2-methylserine and sarcosine were low in FTLD and in other neurodegenerative diseases it has been suggested that abnormalities in these pathways represent an attempt to preserve or restore glycolysis [35]. Spermidine, elevated in FTLD, is a polyamine amino acid that promotes autophagy and has neuroprotective effects in rodent models [36]. The raised levels in FTLD might reflect a response to increased cell death that occurs in patients with the neurodegenerative disease [37]. We found increased serotonin levels in FLTD (FC $10.71, p<0.001)$. Central nervous serotonergic pathways are abnormal in FTLD [18] and serotonin reuptake inhibitors have been used as a symptomatic treatment in FTLD [19]. However, there is usually a limited exchange of serotonin across the blood brain barrier, and the significance of this peripheral serotonin result is unclear for the central nervous system. Peripheral serotonin effects include glucose regulation via its action on pancreatic beta cells, hepatocytes and adipose tissue [38]. Abnormal peripheral serotonin levels in FTLD may therefore again relate to abnormal glucose regulation.

Lipid pathways were also abnormal in FTLD with alterations in several phospholipid, glycerolipid and sphingolipid metabolites. These are important components of cell membranes. Phospholipid pathway metabolites, including phosphatidylserines $(\mathrm{FC} 7-8, p<0.001)$ and 
Fig. 4 Kaplan-Meir Survival Curve of loadings on component 3. Patients were separated into three groups based on their loading onto component 3 . High loading patients had a $z$ score greater than 1, medium between 1 and -1 and low had a $z$ score less than -1 . There was a significant difference in survival curves between the three groups (log rank $p=0.04)$. Graph generated using MatSurv (https:// github.com/aeberg1/MatSurv)

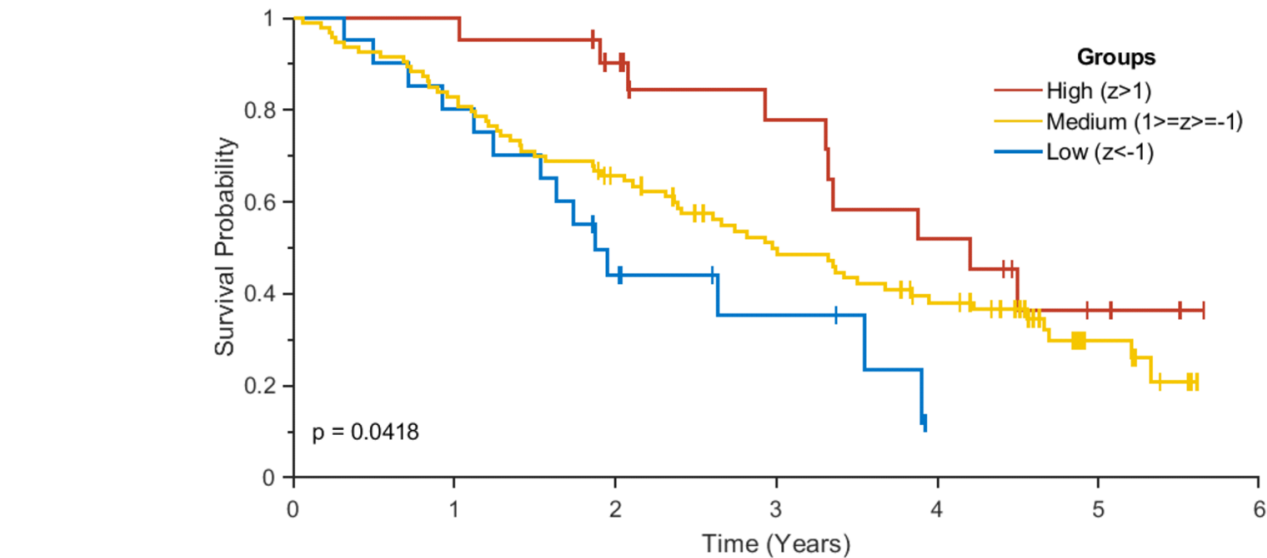

Number at risk

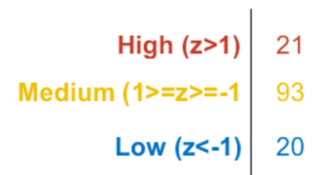

17

58

8
8
27
0 0 phosphoethanolamine (FC 2.61, $p<0.001$ ), showed the greatest differences in FTLD compared to controls. Our results contrast with a lipidomics study of bvFTD which found the same phospholipids were reduced in bvFTD. However, the apparent discrepancy could be explained by the differences in disease stages. Phospholipids are a major component of cell membranes and phosphatidylserine has been proposed as a pro-apoptotic marker in pre-clinical neuronal models of tauopathies $[16,17]$. Sphingosine and its derivative sphingoamine, important components of sphingolipid metabolism, were also elevated in FTLD syndromes. Sphingosine derived lipids comprise up to one third of cell membranes and are highly prevalent in the central nervous system white matter. Dysregulated sphingomyelin metabolism has been implicated in neurodegeneration due to Alzheimer's disease [39] and has been suggested as a potential blood biomarker [40].

Our second aim was to determine whether the metabolome could be used to classify patients by syndrome and provide proof-of-concept for a blood-based biomarker. Classification accuracy, using only the metabolite principal components, was high (88-97\%) between each FTLD syndrome and controls. Sequential feature selection found that only a small subset of components was required, without loss of accuracy. Interestingly classification accuracy did not reflect the strength of the published clinicopathological correlations in each syndrome. Frontotemporal lobar degeneration syndromes are associated with different underlying pathologies, including FTLD-tau and FTLD-TDP43 [1]. Each FTLD syndrome has a different clinicopathological accuracy; the clinical syndrome of PSP-Richardson's syndrome is almost always caused by 4-repeat tau pathology [26] and had a classification accuracy of $95 \%$. BvFTD, which can be caused by
Tau-, TDP43- or FUS-pathology [24], still had a metabolomics accuracy of $96.5 \%$. CBS has poor clinic-pathological correlation and may be associated with corticobasal degeneration, Alzheimer's disease pathology, PSP or other pathology [41], but the syndrome still manifested a metabolomic classification accuracy of $95.6 \%$. This would suggest some of the classifying features results are not neuropathologically specific but rather reflect generalised aspects of progressive neurodegeneration or the widespread physiological stresses that follow.

Classification accuracy was lower between the different FTLD syndromes. This is expected in view of the closely overlapping clinical features and underlying neuropathologies across FTLD syndromes. We suggest that the FTLD syndromes with the closest overlap in phenotype and pathology have the lowest classification accuracy. For example, nfvPPA can be the initial presenting syndrome of PSPpathology or corticobasal degeneration, and nfvPPA can evolve towards a CBS-like phenotype, or CBS-NAV [27, 42-44]. PSP and CBS were weakly differentiated, and share many similar features in pathology and syndrome, as indicated by the nosological status of PSP-CBS and CBS-PSP [26, 27].

Our third aim was to find a prognostic biomarker in FTLD. Component 3 was associated with survival (days to death), independent of disease group, age or gender. A range of metabolic pathways contributed to this component, including phospholipid, amino acid, carbohydrate and cofactor pathways. This suggests the metabolomics marker of mortality risk reflects a signature of underlying progressive neurodegeneration, as opposed to an isolated metabolic pathway alteration. We suggest that the component reflects both environmental and endogenous changes, but further 
studies are required to target the biochemicals comprising component 3. Despite the uncertainty over the causes of the metabolomic differences, our findings suggest that bloodbased biomarkers have potential as diagnostic biomarkers. To confirm the role of metabolomics as a prognostic biomarker longitudinal measures are essential, and comparisons against other differential diagnostic groups such as Parkinson's disease and non-degenerative causes of late-life behavioural change.

Our study has several limitations. Metabolomics can be highly sensitive to differences in sampling, storage and analysis. For practical reasons, and with a view to utility in healthcare settings, our samples were taken at variable times of day, and participants were not fasted. For ethical reasons, no medications were withheld or altered in participants for the purposes of the study. Some participants were taking levodopa or selective serotonin reuptake inhibitors for example. This might account for some of the differences between disease groups and controls. However, to mitigate this risk, we removed metabolites and sub-pathways that have been associated with these medications in reference datasets. We also acknowledge that the Metabolon analysis pipeline cannot differentiate between optical isomers of a metabolite, which may have different physiological properties. Our sample size is modest, we restricted our classification sample sizes to prevent inequalities in the group sizes (which may otherwise bias classifiers). Our sample was therefore limited by the prevalence of the least common disorder. Nonetheless, for small sizes, the $k$-fold cross-validation approach provides a minimally biased estimate of the potential accuracy of classification. For each disease group, we used within-sample cross validation, separating training and tests data on each iteration, but we have not replicated our findings in an independent cohort. Out-of-sample cross-validation was found across the four separate disease groups for component 3 , which was most closely associated with survival. However, further work is required to replicate the findings in other disease-specific cohorts to confirm the utility of metabolomics as a diagnostic biomarker. In anticipation of clinical utility, we focussed on comparison and classification by the syndrome. However, genetic FTD cohorts and retrospective analysis samples from people with post mortem diagnostic confirmation would enable the additional metabolomics analysis by pathology rather than syndrome.

In summary, our findings highlight the widespread metabolic changes in each of four major clinical disorders associated with frontotemporal lobar degeneration. We found that the metabolite profile can be used to classify between FTLD and healthy controls with high accuracy and relate to prognosis. Several metabolites show promise as diagnostic and prognostic biomarkers which if developed could enrich case identification in healthcare settings and in future clinical trials. Further work is required to replicate these findings and test their utility in differentiating between FTLD and pathologically distinct, but phenotypically similar syndromes.

Acknowledgements We are grateful to Dr Matthew Davey, University of Cambridge for advice on statistical procedures and Dr Edward Karolyn from Metabolon Inc for his advice on the Metabolon analysis pipeline

Funding This work was supported by the Holt Fellowship, the Wellcome Trust (103838), the Cambridge Centre for Parkinson-plus, the British Academy (PF160048) and the National Institute for Health Research (NIHR) Cambridge Biomedical Research Centre Dementia and Neurodegeneration Theme (146281).

\section{Compliance with ethical standards}

Conflicts of interest Alexander Murley, P Simon Jones, Ian Coyle Gilchrist, Lucy Bowns, Julie Wiggins and Kamen A. Tsvetanov report no disclosures. James B Rowe reports consultancy for Asceneuron and UCB; research grants from Janssen, AZ-Medimmune, Lilly; and serves as editor for Brain.

Ethical approval This study received ethical approval from the Cambridge Central Research Ethics Committee (12/EE/0475 and 15/ EE/0270) and was carried out in accordance with the ethical standards laid down in the 1964 Declaration of Helsinki and its later amendments.

Informed consent Participants with capacity gave written informed consent. The participation of those who lacked capacity was discussed with a personal consultee in accordance with the Mental Capacity Act (UK).

Open Access This article is licensed under a Creative Commons Attribution 4.0 International License, which permits use, sharing, adaptation, distribution and reproduction in any medium or format, as long as you give appropriate credit to the original author(s) and the source, provide a link to the Creative Commons licence, and indicate if changes were made. The images or other third party material in this article are included in the article's Creative Commons licence, unless indicated otherwise in a credit line to the material. If material is not included in the article's Creative Commons licence and your intended use is not permitted by statutory regulation or exceeds the permitted use, you will need to obtain permission directly from the copyright holder. To view a copy of this licence, visit http://creativecommons.org/licenses/by/4.0/.

\section{References}

1. MacKenzie IRA, Neumann M, Bigio EH et al (2010) Nomenclature and nosology for neuropathologic subtypes of frontotemporal lobar degeneration: an update. Acta Neuropathol 119:1-4. https:// doi.org/10.1007/s00401-009-0612-2

2. Riedl L, Mackenzie IR, Förstl H et al (2014) Frontotemporal lobar degeneration: current perspectives. Neuropsychiatr Dis Treat 10:297-310. https://doi.org/10.2147/NDT.S38706

3. Lewitt PA, Li J, Lu M et al (2017) Metabolomic biomarkers as strong correlates of Parkinson disease progression. Neurology 88:862-869. https://doi.org/10.1212/WNL.0000000000003663

4. Toledo JB, Arnold M, Kastenmüller G et al (2017) Metabolic network failures in Alzheimer's disease: a biochemical road map. 
Alzheimer's Dement 13:965-984. https://doi.org/10.1016/j. jalz.2017.01.020

5. Mastrokolias A, Pool R, Mina E et al (2016) Integration of targeted metabolomics and transcriptomics identifies deregulation of phosphatidylcholine metabolism in Huntington's disease peripheral blood samples. Metabolomics 12:1-15. https://doi. org/10.1007/s11306-016-1084-8

6. Eller M, Williams DR (2009) Biological fluid biomarkers in neurodegenerative parkinsonism. Nat Rev Neurol 5:561-570. https:// doi.org/10.1038/nrneurol.2009.135

7. Murley AG, Coyle-Gilchrist I, Rouse M et al (2019) Redefining the multidimensional clinical phenotypes of frontotemporal lobar degeneration syndromes. Brain (in press)

8. Rohrer JD, Lashley T, Schott JM et al (2011) Clinical and neuroanatomical signatures of tissue pathology in frontotemporal lobar degeneration. Brain 134:2565-2581. https://doi.org/10.1093/brain /awr198

9. Ferrari R, Hernandez DG, Nalls MA et al (2014) Frontotemporal dementia and its subtypes: a genome-wide association study. Lancet Neurol 13:686-699. https://doi.org/10.1016/S1474 -4422(14)70065-1

10. Ferrari R, Grassi M, Salvi E et al (2015) A genome-wide screening and SNPs-to-genes approach to identify novel genetic risk factors associated with frontotemporal dementia. Neurobiol Aging 36:2904.e13-2904.e26. https://doi.org/10.1016/j.neurobiola ging.2015.06.005

11. Diekstra FP, Van Deerlin VM, Van Swieten JC et al (2014) C9orf72 and UNC13A are shared risk loci for amyotrophic lateral sclerosis and frontotemporal dementia: a genome-wide meta-analysis. Ann Neurol 76:120-133. https://doi.org/10.1002/ana.24198

12. Höglinger GU, Melhem NM, Dickson DW et al (2011) Identification of common variants influencing risk of the tauopathy progressive supranuclear palsy. Nat Genet 43:699-705. https://doi. org/10.1038/ng.859

13. Melquist S, Craig DW, Huentelman MJ et al (2007) Identification of a novel risk locus for progressive supranuclear palsy by a pooled genomewide scan of 500,288 single-nucleotide polymorphisms. Am J Hum Genet 80:769-778. https://doi. org/10.1086/513320

14. Laakso MP, Jukarainen NM, Vepsäläinen J (2015) Diagnosis of dementias by high-field 1H MRS of cerebrospinal fluid. J Neurol Neurosurg Psychiatry 86:1286-1290. https://doi.org/10.1136/ jnnp-2015-311171

15. Kim WS, Jary E, Pickford R et al (2018) Lipidomics analysis of behavioral variant frontotemporal dementia: a scope for biomarker development. Front Neurol 9:1-11. https://doi.org/10.3389/fneur .2018.00104

16. Segawa K, Nagata S (2015) An apoptotic "Eat Me" signal: phosphatidylserine exposure. Trends Cell Biol 25:639-650. https://doi. org/10.1016/j.tcb.2015.08.003

17. Brelstaff J, Tolkovsky AM, Ghetti B et al (2018) Living neurons with tau filaments aberrantly expose phosphatidylserine and are phagocytosed by microglia. Cell Rep 24:1939-1948.e4. https:// doi.org/10.1016/j.celrep.2018.07.072

18. Murley AG, Rowe JB (2018) Neurotransmitter deficits from fronto temporal lobar degeneration. Brain 141:1263-1285. https://doi. org/10.1093/brain/awx327

19. Hughes LE, Rittman T, Regenthal R et al (2015) Improving response inhibition systems in frontotemporal dementia with citalopram. Brain 138:1961-1975. https://doi.org/10.1093/brain/ awv133

20. Rascovsky K, Hodges JR, Kipps CM et al (2007) Diagnostic criteria for the behavioral variant of frontotemporal dementia (bvFTD): current limitations and future directions. Alzheimer Dis Assoc Disord 21:S14-S18. https://doi.org/10.1097/WAD.0b013 e31815c3445
21. Ahmed RM, Irish M, Henning E et al (2016) Assessment of eating behavior disturbance and associated neural networks in frontotemporal dementia. JAMA Neurol 73:282-290. https://doi. org/10.1001/jamaneurol.2015.4478

22. González Sánchez JJ, Nora JE, De Notaris M et al (2010) A case of malignant monophasic multiple sclerosis (Marburg's disease type) successfully treated with decompressive hemicraniectomy. J Neurol Neurosurg Psychiatry 81:1056-1057. https://doi. org/10.1136/jnnp.2007.142133

23. Ahmed RM, Landin-Romero R, Collet TH et al (2017) Energy expenditure in frontotemporal dementia: a behavioural and imaging study. Brain 140:171-183. https://doi.org/10.1093/brain/ aww263

24. Rascovsky K, Hodges JR, Knopman D et al (2011) Sensitivity of revised diagnostic criteria for the behavioural variant of frontotemporal dementia. Brain 134:2456-2477. https://doi. org/10.1093/brain/awr179

25. Gorno-Tempini ML, Hillis AE, Weintraub S et al (2011) Classification of primary progressive aphasia and its variants. Neurology 76:1006-1014. https://doi.org/10.1212/WNL.0b013e3182 $1103 \mathrm{e} 6$

26. Höglinger GU, Respondek G, Stamelou M et al (2017) Clinical diagnosis of progressive supranuclear palsy: the movement disorder society criteria. Mov Disord 32:853-864. https://doi. org/10.1002/mds.26987

27. Armstrong MJ, Litvan I, Lang AE et al (2013) Criteria for the diagnosis of corticobasal degeneration. Neurology 80:496-503. https://doi.org/10.1212/WNL.0b013e31827f0fd1

28. Evans AM, DeHaven CD, Barrett T et al (2009) Integrated, nontargeted ultrahigh performance liquid chromatography/electrospray ionization tandem mass spectrometry platform for the identification and relative quantification of the small-molecule complement of biological systems. Anal Chem 81:6656-6667. https://doi.org/10.1021/ac901536h

29. van den Berg RA, Hoefsloot HCJ, Westerhuis JA et al (2006) Centering, scaling, and transformations: improving the biological information content of metabolomics data. BMC Genom 7:1-15. https://doi.org/10.1186/1471-2164-7-142

30. Molinaro AM, Simon R, Pfeiffer RM (2005) Prediction error estimation: a comparison of resampling methods. Bioinformatics 21:3301-3307. https://doi.org/10.1093/bioinformatics/bti499

31. Chang CC, Lin CJ (2011) LIBSVM: a library for support vector machines. ACM Trans Intell Syst Technol 2:1-27. https://doi. org/10.1145/1961189.1961199

32. Diehl-Schmid J, Grimmer T, Drzezga A et al (2007) Decline of cerebral glucose metabolism in frontotemporal dementia: a longitudinal 18F-FDG-PET-study. Neurobiol Aging 28:42-50. https ://doi.org/10.1016/j.neurobiolaging.2005.11.002

33. Foster NL, Gilman S, Berent S et al (1988) Cerebral hypometabolism in progressive supranuclear palsy studied with positron emission tomography. Ann Neurol 24:399-406. https://doi. org/10.1002/ana.410240308

34. Hosaka K, Ishii K, Sakamoto S et al (2002) Voxel-based comparison of regional cerebral glucose metabolism between PSP and corticobasal degeneration. J Neurol Sci 199:67-71. https://doi. org/10.1016/S0022-510X(02)00102-8

35. Wilkins JM, Trushina E (2018) Application of metabolomics in Alzheimer's disease. Front Neurol 8:1-20. https://doi.org/10.3389/ fneur.2017.00719

36. Madeo F, Eisenberg T, Pietrocola F, Kroemer G (2018) Spermidine in health and disease. Science (80-) 359:eaan2788. https:// doi.org/10.1126/science.aan2788

37. Stefanis L, Burke RE, Greene LA (1997) Apoptosis in neurodegenerative disorders. Curr Opin Neurol 10:299-305. https://doi. org/10.1097/00019052-199708000-00004 
38. El-Merahbi R, Löffler M, Mayer A, Sumara G (2015) The roles of peripheral serotonin in metabolic homeostasis. FEBS Lett 589:1728-1734. https://doi.org/10.1016/j.febslet.2015.05.054

39. Haughey NJ, Bandaru VVR, Bae M, Mattson MP (2010) Roles for dysfunctional sphingolipid metabolism in Alzheimer's disease neuropathogenesis. Biochim Biophys Acta Mol Cell Biol Lipids 1801:878-886. https://doi.org/10.1016/j.bbalip.2010.05.003

40. Mielke MM, Haughey NJ (2012) Could plasma sphingolipids be diagnostic or prognostic biomarkers for Alzheimer's disease? Clin Lipidol 7:525-536. https://doi.org/10.2217/clp.12.59

41. Alexander SK, Rittman T, Xuereb JH et al (2014) Validation of the new consensus criteria for the diagnosis of corticobasal degeneration. J Neurol Neurosurg Psychiatry 85:923-927. https://doi. org/10.1136/jnnp-2013-307035
42. Rohrer JD, Paviour D, Bronstein AM et al (2010) Progressive supranuclear palsy syndrome presenting as progressive nonfluent aphasia: a neuropsychological and neuroimaging analysis. Mov Disord 25:179-188. https://doi.org/10.1002/mds.22946

43. Rohrer JD, Rossor MN, Warren JD (2010) Apraxia in progressive nonfluent aphasia. J Neurol 257:569-574. https://doi.org/10.1007/ s00415-009-5371-4

44. Coyle-Gilchrist ITS, Dick KM, Patterson K et al (2016) Prevalence, characteristics, and survival of frontotemporal lobar degeneration syndromes. Neurology 86:1736-1743. https://doi. org/10.1212/WNL.0000000000002638 